\title{
Charge shelving and bias spectroscopy for the readout of a charge qubit on the basis of superposition states.
}

\author{
Andrew D. Greentre, ${ }^{1,2}$ A. R. Hamilton, ${ }^{1}$ and F. Green ${ }^{1}$ \\ ${ }^{1}$ Centre for Quantum Computer Technology, School of Physics, \\ The University of New South Wales, Sydney, NSW 2052, Australia \\ ${ }^{2}$ Centre for Quantum Computer Technology, School of Physics, \\ The University of Melbourne, Melbourne, Victoria 3010, Australia
}

(Dated: 16 July 2004)

\begin{abstract}
Charge-based qubits have been proposed as fundamental elements for quantum computers. One commonly proposed readout device is the single-electron transistor (SET). SETs can distinguish between localized charge states, but lack the sensitivity to directly distinguish superposition states, which have greatly enhanced coherence times compared with position states. We propose introducing a third dot, and exploiting energy dependent tunnelling from the qubit into this dot (bias spectroscopy) for pseudo-spin to charge conversion and superposition basis readout. We introduce an adiabatic fast passage-style charge pumping technique which enables efficient and robust readout via charge shelving, avoiding problems due to finite SET measurement time.

PACS numbers: 73.21.La, 03.67.Lx, 73.23.Hk
\end{abstract}

The experimental observation, manipulation and utilization of coherent quantum mechanical properties in solid-state systems are key technological challenges for this century. The importance of incoherent quantum properties has been essential for the development of microelectronics and it is hoped that coherent quantum effects will spawn new technologies including, but not necessarily limited to, quantum computers 1 .

In the development of coherent solid-state systems compatible with quantum computing, superconducting systems have a clear advantage due to the presence of macroscopic quantum states and key milestones have already been reached ${ }^{2.3 .4}$. Coherent transport in semiconductor two-dimensional electron-gas (2DEG) systems has been observed,,$\frac{5}{\underline{5}}$ and recently a charge qubit has been realized in a GaAs double $\operatorname{dot}^{\underline{6}}$. Despite this, there is a strong impetus to develop coherent technologies that are compatible with the semiconductor industry, especially those based on silicon-metal-oxide technology, owing to its mature manufacturing technology and potential scalability advantages 7.8 .9 .

Of particular interest are charge-based quantum computers $\frac{10}{10}$, for example in Cooper-pair box arrangements ${ }^{2.11}$, and semiconductor systems ${ }^{8.9 .12}$, because of the relative ease of readout using high sensitivity electrometers. One such electrometer is the radio-frequency single-electron transistor (rf-SET) ${ }^{13}$, which has been shown to be compatible with quantum computing requirements ${ }^{14}$. The relative ease of coupling to a charge-based qubit is, however, also responsible for giving the qubit a short decoherence time, as the charge distribution of the qubit couples readily to the local electrostatic environment. By operating a charge qubit at the degeneracy point, the natural basis is the superposition basis. These states couple less strongly to the electrostatic environment, so the decoherence rate of a superposition basis qubit should be much less than that for a position basis qubit. Conventional electrometers lack the sensitivity to directly distinguish between superposition states. Our scheme provides a robust mechanism to convert information from the superposition basis to an accessible position basis, incorporating charge shelving in a fashion not available with conventional double-dot schemes.

We consider a coherent triple-dot, one-electron system, with a strongly-coupled qubit, and a weakly-coupled 'probe' dot, illustrated in Fig. [1] Varying the energy of the third dot, relative to the qubit, achieves a form of bias spectroscopy, reminiscent of the optical Autler-Townes (AT) experiment ${ }^{15}$. Such spectroscopy serves to probe the system dynamics. However for single-shot qubit readout we propose an adiabatic fast passage $(\mathrm{AFP})^{16}$ like process to perform superposition-to-position state pumping. This constitutes a form of charge-shelving and is analogous to a scheme for adiabatic transport in a

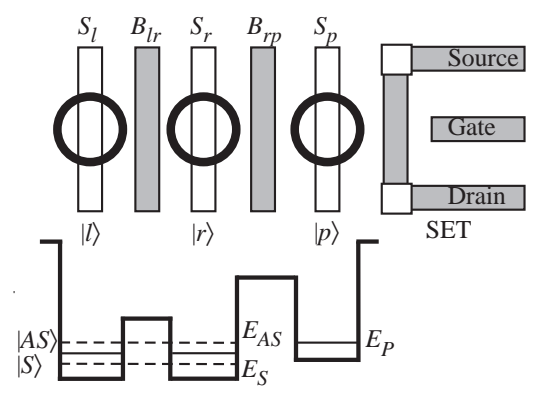

FIG. 1: (Top) configuration showing phosphorus donors and gate structure. There is one electron and an SET for readout. The qubit is defined by donors marked $|l\rangle$ and $|r\rangle$, monitoring the third donor, $|p\rangle$ (probe) provides the readout. State energies $(E)$ are controlled via gates $S_{l}, S_{r}$, and $S_{p}$, and the coherent tunnelling rates, $\Omega_{l r}$ and $\Omega_{r p}$ are controlled by barrier gates $B_{l r}$ and $B_{r p}$ respectively. (Bottom) triple well diagram with $E_{l}=E_{r}$, the symmetric $|S\rangle$ and antisymmetric $|A S\rangle$ states are equally separated from $E_{l}$. $E_{p}$ has been tuned to $E_{A S}$ so resonant tunnelling between $|A S\rangle$ and $|p\rangle$ occurs. 
double-dot system by Brandes and Vorrath ${ }^{17}$. Although our scheme should be applicable to any three-state, oneelectron system, for clarity we focus on the phosphorusin-silicon system of Hollenberg et $a l^{9}$. We note that subsequent to our initial suggestion, a similar scheme has been realized in a beautiful experiment by Astafiev et $a l^{18}$ for a Josephson charge qubit.

Our scheme requires a small increase in the complexity required to perform a charge-qubit measurement over simpler two-donor schemes. Therefore we must identify the circumstances where it will be advantageous.

For any quantum computing scheme there are many important timescales, and the choice of an effective measurement scheme depends on the relative values of each. There are the two environmental dephasing times, $T_{1}$ and $T_{2}$ corresponding to population and coherence relaxation respectively. $\tau_{\text {meas }}$ is the time required for a measurement to occur, and because a measurement projects the system into a basis state of the measurement device, this contributes an effective $T_{2}$. We define $\tau_{\text {osc }}$ as the time for one coherent oscillation to occur and $\tau_{\text {gate }}^{-1}$ as the maximum rate at which signals can be sent to manipulate the qubit. For a functioning qubit we must have $\tau_{\text {osc }}, \tau_{\text {gate }} \ll T_{1}, T_{2}$, and this is assumed in our discussion.

Nondestructive single-shot readout requires $\tau_{\text {meas }} \ll$ $T_{1}$. $T_{1}$ is a function of the energy separation between states, and for good charge localization (necessary for SET readout) we must operate far from degeneracy, i.e. where $T_{1}$ is minimized. Recent experiments and analysis of $\mathrm{rf}-\mathrm{SETs}^{14}$ suggest that if the induced SET island charge is $\sim 0.01 \mathrm{e}$, it will take $\tau_{\text {meas }} \sim 2 \mu$ s to achieve an error rate of 0.1 . This $\tau_{\text {meas }}$ means that although proof of principle experiments using signal averaging will be possible, single shot measurements for readout and error correction in a practical device will be problematic as the population will decay faster than it can be measured. By introducing charge shelving to isolate the charge at the bias position that maximizes $T_{1}$ during readout, we ameliorate this.

To exploit SET readout and superposition basis operation, we must transfer quantum information from the superposition basis, to a position basis. This may be done either nonadiabatically or adiabatically.

Nonadiabatic operations, which could be performed in either double or triple dot geometries, require $\tau_{\text {gate }} \ll$ $T_{1}, T_{2}$ and $\tau_{\text {osc }}$. If sufficient bandwidth is available they constitute the fastest mechanism for transferring population. Nonadiabatic operations are extremely sensitive to noise and gate errors, and hence attention has turned to adiabatic methods.

There are at least two adiabatic timescales, the first being the usual quasi-static case where $\tau_{\text {gate }} \gg T_{1}, T_{2}, \tau_{\text {osc }}$. Adiabatic transfer based on quasi-static operations is conceptually easy and applicable to both double and triple dot schemes. By necessity such manipulations are slow, and there may be incompatibility between $\tau_{\text {gate }}$ and $T_{1}$, meaning such rotations are not suitable for quantum computer readout. Triple-dot systems afford the possibil-

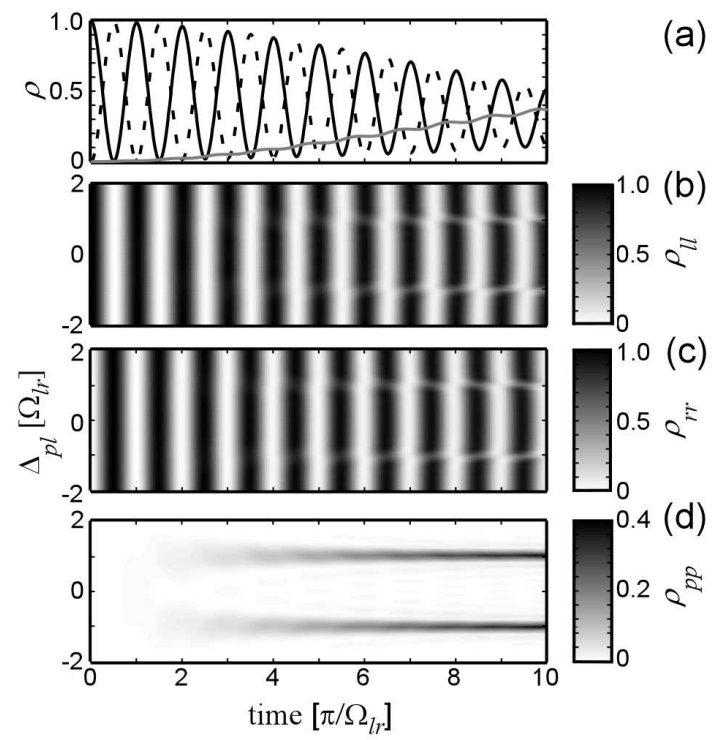

FIG. 2: (a) $\rho_{l l}$ (black solid line), $\rho_{r r}$ (black broken line) and $\rho_{p p}$ (grey solid line) as a function of time (in units of $\pi / \Omega_{l r}$ ) for $\Delta_{p l}=\Omega_{l r}=2$. Density plots showing $\rho_{l l}$ (b), $\rho_{r r}$ (c), and $\rho_{p p}$ (d), as a function of time and $\Delta_{p l}$ (in units of $\Omega_{l r}$ ) for $E_{l}=E_{r}=0$, $\Omega_{r p}=\Omega_{l r} / 20$ and $\Gamma=\Omega_{l r} / 100$ for a qubit in state $|l\rangle$ at time $t=0$. Note the dominant oscillatory behavior in $\rho_{l l}(t)$ and $\rho_{r r}(t)$ and the AT doublet-like feature in $\rho_{p p}$.

ity for a further adiabatic timescale, where adiabatic passage techniques exploiting appropriate parameter modulation, allow decoupling of the adiabatic pathway from the dephasing times ${ }^{16}$, i.e. $\tau_{\text {osc }} \ll \tau_{\text {gate }} \ll T_{1}, T_{2}$. It is this second adiabatic timescale that we exploit for AFP charge-shelving. For a more complete discussion of timescales in coherently driven systems see Ref 19 .

To summarize, any practical qubit requires $\tau_{\text {osc }}, \tau_{\text {gate }}, \tau_{\text {meas }} \ll T_{2}, T_{1}$, and most implementations proposed to date also require $\tau_{\text {gate }} \ll \tau_{\text {osc }}$. Our scheme can function with $\tau_{\text {gate }}>\tau_{\text {osc }}$, to identify the signature of coherent oscillations, and charge-shelving scheme provides a mechanism to increase $T_{1}$ to ensure that $\tau_{\text {meas }} \ll T_{1}$. AFP provides the advantages of adiabatic control without restricting gate operations to the timescales for decoherence rates. Even so, it is necessary to explore any given implementation fully to determine if our scheme will provide a tangible advantage.

One further consideration is that access to multiple bases is necessary for state tomography ${ }^{20}$, which is important for qubit characterization. The extra freedom afforded by the triple-donor system suggests that a hybrid position/superposition readout system may be realizable which would have advantages for tomography and we will investigate this possibility elsewhere.

The three-donor system is shown in Fig. 1] with three ionized phosphorus donors (open circles) sharing a single electron. A strongly coupled qubit is defined by donors $l$ (left) and $r$ (right). The weakly coupled probe is labelled $p$. We follow the gate notation used in Refs ${ }^{9,12}$. The energies of each single-electron state are controlled using 


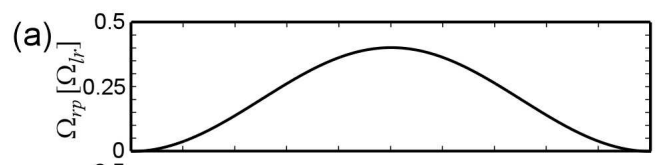

(b)

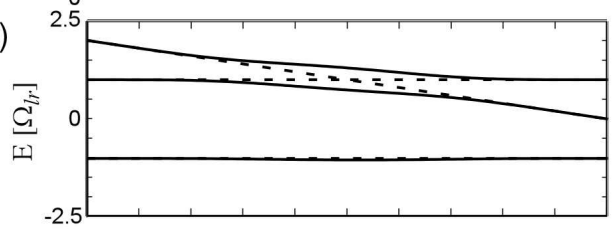

(c)

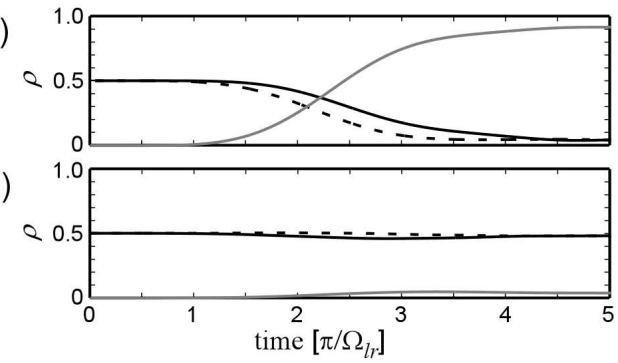

FIG. 3: AFP readout (a) $\Omega_{r p}$ as a function of time (in units of $\left.\pi / \Omega_{l r}\right)$ showing modulation of the coherent tunnelling rate. (b) State energies (in units of $\Omega_{l r}$ ) as a function of time. The dashed, black lines represent the energies unperturbed by $\Omega_{r p}$, whilst the solid blue lines represent state energies with $\Omega_{r p}(t)$ applied. At $t=0$ the states are (highest to lowest energy) $|p\rangle,|A S\rangle$ and $|S\rangle$. (c),(d) $\rho_{l l}$ (black solid line), $\rho_{r r}$ (black broken line) and $\rho_{p p}$ (grey solid line) as a function of time for $\rho(0)=|A S\rangle$ (c) $\rho(0)=|S\rangle$ (d). In all cases $\Omega_{r p}^{\max }=0.4 \Omega_{l r}$.

shift gates (open rectangles), $S$, and the energies of these states are $E_{l}, E_{r}, E_{p}$, with $\Delta_{\alpha, \beta}=E_{\alpha}-E_{\beta}, \alpha, \beta=l, r, p$. Coherent tunnelling is controlled by barrier gates (grey rectangles), which vary the barrier height. The barrier gate between donors $l$ and $r(r$ and $p)$ is labelled $B_{l r}$ $\left(B_{r p}\right)$. We ignore direct tunnelling between $l$ and $p$. An SET reads out the electron on $p$. The natural basis for the qubit will be the superposition basis, we write the symmetric (anti-symmetric) state as $|S\rangle=(1 / \sqrt{2})(|l\rangle+$ $|r\rangle)(|A S\rangle=(1 / \sqrt{2})(|l\rangle-|r\rangle))$.

We vary $E_{p}$ and monitor the population in $p$ with the SET, observing nonzero populations in $p$ only when $E_{p}$ is quasi-degenerate with either of the superposition states. Similar bias spectroscopy is seen in open 2DEG systems ${ }^{5}$. In optical AT experiments, it is more usual to monitor the response of a weak probe field, which is proportional to the coherence $\rho_{r p}$.

We solve the density matrix equations of motion to give both transient and spectroscopic insight into the dynamics. This is similar to the approach in Ref ${ }^{19}$. We express the coherent tunnelling rate (analogous to the Rabi frequency in optical experiments) as $\Omega_{\alpha \beta}=2 \pi / \tau_{\text {osc }}^{\alpha \beta}$, where the $B$ gate dependance has been suppressed. This follows the spirit of Gurvitz's ${ }^{21}$ treatment for the two dot system and that of Renzoni and Brandes 22 in a triple-well system. The Hamiltonian is

$$
\begin{aligned}
\mathcal{H}= & \Delta_{r l}|r\rangle\left\langle r\left|+\Delta_{p l}\right| p\right\rangle\langle p| \\
& -\hbar \Omega_{l r}(|l\rangle\langle r|+| r\rangle\langle l|)-\hbar \Omega_{r p}(|r\rangle\langle p|+| p\rangle\langle r|) .
\end{aligned}
$$

This Hamiltonian is identical to an optically driven threelevel atom in the rotating-wave approximation 23 .

The density matrix equations of motion are written

$$
\dot{\rho}=-(i / \hbar)[\mathcal{H}, \rho]+\mathcal{L},
$$

with $\rho$ the density matrix and $\mathcal{L}$ a dephasing operator. $T_{2}$ processes are modelled by a dephasing rate $\Gamma$ which is assumed affect all coherences, $T_{1}$ processes are described by rates of incoherent population transfer ${ }^{24}$

$$
\Gamma_{\alpha \beta}=\chi_{\alpha \beta} \Delta_{\alpha \beta} /\left[1-\exp \left(\Delta_{\alpha \beta} / k T\right)\right],
$$

where $k$ is Boltzmann's constant, $T$ the temperature and $\chi_{\alpha \beta}$ a rate from the tunnelling probability between $\alpha$ and $\beta$. $\Gamma_{\alpha \beta}$ is the population transfer rate from $\beta$ to $\alpha$.

The density matrix equations of motion are therefore

$$
\begin{aligned}
\dot{\rho}_{l l}= & i \Omega_{l r}\left(\rho_{r l}-\rho_{l r}\right)-\Gamma_{r l} \rho_{l l}+\Gamma_{l r} \rho_{r r} \\
\dot{\rho}_{l r}= & i\left[\frac{\Delta_{r l}}{\hbar} \rho_{l r}+\Omega_{l r}\left(\rho_{r r}-\rho_{l l}\right)-\Omega_{r p} \rho_{l p}\right]-\Gamma \rho_{l r} \\
\dot{\rho}_{l p}= & i\left[\frac{\Delta_{p l}}{\hbar} \rho_{l p}+\Omega_{l r} \rho_{r p}-\Omega_{r p} \rho_{l r}\right]-\Gamma \rho_{l p} \\
\dot{\rho}_{r r}= & i\left[\Omega_{l r}\left(\rho_{l r}-\rho_{r l}\right)+\Omega_{r p}\left(\rho_{p r}-\rho_{r p}\right)\right] \\
& +\Gamma_{r l} \rho_{l l}-\left(\Gamma_{l r}+\Gamma_{p r}\right) \rho_{r r}+\Gamma_{r p} \rho_{p p} \\
\dot{\rho}_{r p}= & i\left[\frac{\Delta_{p r}}{\hbar} \rho_{r p}+\Omega_{r p}\left(\rho_{p p}-\rho_{r r}\right)+\Omega_{l r} \rho_{l p}\right]-\Gamma \rho_{r p}, \\
\dot{\rho}_{p p}= & i \Omega_{r p}\left(\rho_{r p}-\rho_{p r}\right)+\Gamma_{p r} \rho_{r r}-\Gamma_{r p} \rho_{p p}, \\
\rho_{\alpha \beta}= & \rho_{\beta \alpha}^{*} \\
1= & \rho_{l l}+\rho_{r r}+\rho_{p p} .
\end{aligned}
$$

We numerically integrated Eqs. 1 with $\rho_{l l}(0)=1$ to highlight the dynamics. Maximum coherence times require $E_{l}=E_{r}$ which is used in our calculations. For the third dot to act as a weak probe, $\Omega_{r p} \ll \Omega_{l r}$. We have therefore chosen $\Gamma=\Omega_{l r} / 100, \chi_{\alpha \beta}=0$ and $\Omega_{r p}=\Omega_{l r} / 20$. Fig. 2 (a) shows the time dependent populations when $E_{p}=E_{A S}$. The dominant feature is the coherent population oscillations between $l$ and $r$. There is a steady buildup of population in $p$, which is our measurement signal. Figs. 22(b)-(d) show $\rho_{l l}, \rho_{r r}$, and $\rho_{p p}$ respectively, as a function of time and $\Delta_{p r}$. Again, the dominant behavior is the coherent oscillation between $l$ and $r$, however when $\Delta_{p r}= \pm \Omega_{l r}$, resonant tunnelling into $p$ occurs, yielding a doublet in $\rho_{p p}$ similar to the AT doublet. Measurement of probe population is therefore sensitive to the population in the symmetric and antisymmetric modes of the $l-r$ system.

Bias spectroscopy should be useful for characterizing qubit properties, albeit incompatible with readout of a practical quantum computer. This is due to (i) coherent oscillations on the $r-p$ transition reducing readout fidelity, (ii) the need to set and accurately maintain $\Delta_{p l}$ over the measurement time, and (iii) small average populations tunnelling into $p$ requiring multiple experiments. To avoid these problems, we propose a form of chargeshelving that adiabatically drives the population into $|p\rangle$ 
from one of the superposition states, realized by controlling the tunnelling in a fashion related to AFP. The advantages of this are that $\rho_{p p}$ is adiabatically driven to a large value (approaching unity) in a short time (typically a few $\Omega_{l r}^{-1}$ ), with robustness to gate errors. Because of the energy dependence of $T_{1}$, it is most useful to perform AFP between state $|p\rangle$ and the more energetic of the two superposition states, i.e. $|A S\rangle$. One must take care in choosing the modulation trajectory for $|p\rangle$ in this case, as there will be some off-resonant interactions. Our results are promising however, and further optimization can be done.

The trajectory taken by state $|p\rangle$ is governed by both $\Delta_{p l}$ and $\Omega_{r p}$ and for the traces in Figs. 3] they were:

$$
\begin{aligned}
\Delta_{l p} & =2 \Omega_{l r}\left(1-t / t_{\max }\right), \\
\Omega_{r p} & =\Omega_{r p}^{\max }\left[1-\cos \left(2 \pi t / t_{\max }\right)\right] / 2,
\end{aligned}
$$

where $\Omega_{r p}^{\max }=0.4 \Omega_{l r}, t_{\min }=0$ and $t_{\max }=5 \pi / \Omega_{l r}$. In order to make this more explicit, Fig. 3 (a) shows $\Omega_{r p}(t)$, in keeping with conventional AFP schemes, our scheme is fairly insensitive to the exact form of $\Omega_{r p}$; and (b) is a diagram showing the energy levels as a function of time.

Fig. 3. (c) shows the populations for the qubit being initially prepared in $|A S\rangle$. After AFP most of the population has been driven into $|p\rangle$. Similarly Fig. 3 (d) shows the effect of the AFP trajectory on $|S\rangle$, in this case there is minimal population transfer. We are presently performing more detailed numerical experiments to optimize the population transfer and examine tomographic applications of the scheme, as foreshadowed above. One important issue is the potential re-initialization of the qubit after readout. The AFP scheme as presented is entirely time reversible, and therefore one can simply reverse the scheme to pump an electron from the probe state into the anti-symmetric state to reset the qubit.

In summary, we have presented a scheme for performing bias spectroscopy on a qubit, where resonant tunnelling from the superposition states to a third dot is read out with a SET. Such a scheme is a solid-state analog of the optical Autler-Townes scheme. Using bias spectroscopy, one can map out the energy-level space and this may prove a useful probe of coherent coupling where conventional electrometers are unable to resolve the dynamics. Because the bias spectroscopy described here should be able to resolve arbitrary energy differences, this idea may be applied to discriminating between the singlet and triplet states of a two-spin system, such as in Ref 7 . This constitutes an alternate approach to spin readout which we will describe further elsewhere 25 . For useful readout, we propose an AFP style scheme, where population is driven into the probe state. This has the advantage of high fidelity readout with robustness to gate errors. It also introduces a form of charge-shelving, avoiding problems due to measurement times long compared with $T_{1}$.

The authors would like to thank L. C. L. Hollenberg (University of Melbourne) and H. S. Goan (University of Queensland) for useful discussions. This work was supported by the Australian Research Council, the Australian government and by the US National Security Agency (NSA), Advanced Research and Development Activity (ARDA) and the Army Research Office (ARO) under contract number DAAD19-01-1-0653.
1 M. A. Nielsen and I. L. Chuang, Quantum Computation and Quantum Information (Cambridge University Press, Cambridge, England 2000).

2 Y. Nakamura, Yu. A. Pashkin, and J. S. Tsai, Nature 398, 786 (1999).

3 D. Vion, A. Aassime, A. Cottet, P. Joyez, H. Pothier, C. Urbina, D. Esteve, and M. H. Devoret, Science 296, 886 (2002).

4 Yu. A. Pashkin, T. Yamamoto, O. Astafiev, Y. Nakamura, D. V. Averin, and J. S. Tsai, Nature 421, 823 (2003).

5 W. G. van der Wiel, S. De Franceschi, J. M. Elzerman, T. Fujisawa, S. Tarucha, and L. P. Kouwenhoven, Rev. Mod. Phys. 75, 1 (2003).

6 T. Hayashi, T. Fujisawa, H. D. Cheong, Y. H. Jeong, and Y. Hirayama, Phys. Rev. Lett. 91, 226804 (2003).

7 B. E. Kane, Nature 393, 133 (1998); B. E. Kane, N. S. McAlpine, A. S. Dzurak, R. G. Clark, G. J. Milburn, H. B. Sun, and H. Wiseman, Phys. Rev. B 61, 2961 (2000).

8 R. G. Clark et al., Philos. Trans. R. Soc. Lond., Ser. A 361, 1451 (2003).

9 L. C. L. Hollenberg, A. S. Dzurak, C. Wellard, A. R. Hamilton, D. J. Reilly, G. J. Milburn, and R. G. Clark, Phys. Rev. B 69, 113301 (2004).

10 A. Ekert and R. Josza, Rev. Mod. Phys. 68, 733 (1996).

11 Yu. Makhlin, G. Schön, A. Shnirman, Nature 398, 786 (1999).

12 S. G. Schirmer, A. D. Greentree, and D. K. L. Oi, quant-ph/0305052 (2003).
13 R. J. Schoelkopf, P. Wahlgren, A. A. Kozhevnikov, P. Delsing, and D. E. Prober, Science 280, 1238 (1998).

14 T. M. Buehler, D. J. Reilly, R. P. Starrett, A. D. Greentree, A. R. Hamilton, A. S. Dzurak, and R. G. Clark, cond-mat/0304384 (2003).

15 S. H. Autler and C. H. Townes, Phys. Rev. 100703 (1955).

16 N. V. Vitanov, T. Halfmann, B. W. Shore, and K. Bergmann, Annu. Rev. Phys. Chem. 52, 763 (2001).

17 T. Brandes and T. Vorrath, Phys. Rev. B 66, 075341 (2002).

18 O. Astafiev, Yu. A. Pashkin, T. Yamamoto, Y. Nakamura, and J. S. Tsai, Phys. Rev. B 69, 180507 (2004).

19 A. D. Greentree, T. B. Smith, S. R. de Echaniz, A. V. Durrant, J. P. Marangos, D. M. Segal, and J. A. Vaccaro, Phys. Rev. A, 65, $053802(2002)$.

20 D. F. V. James, P. G. Kwiat, W. J. Munro, and A. G. White, Phys. Rev. A 64, 052312 (2001).

21 S. A. Gurvitz, Phys. Rev. B 56, 15215 (1997).

22 F. Renzoni and T. Brandes, Phys. Rev. B 64, 245301 (2001).

23 B. W. Shore, The Theory of Coherent Atomic Excitation (Wiley, New York, 1990).

24 G. Tóth, A. O. Orlov, I. Amlani, C. S. Lent, G. H. Bernstein, and G. L. Snider, Phys. Rev. B 60, 16906 (1999).

25 A. D. Greentree, A. R. Hamilton, L. C. L. Hollenberg, and R. G. Clark, cond-mat/0403449 (2004). 